\title{
Is adaptive treatment planning for single-channel vaginal brachytherapy necessary?
}

\author{
Jan-Erik Palmgren, PhLicl, Jan Seppälä, PhD', Maarit Anttila, MD² \\ 'Radiotherapy Department of the Center of Oncology, Kuopio University Hospital, Finland, ${ }^{2}$ Department of Obstetrics and Gynecology, \\ Kuopio University Hospital, Finland
}

\begin{abstract}
Purpose: In vaginal cuff brachytherapy, only limited information is available about the need for individualized treatment planning or imaging. Treatment planning is still performed mostly with no contouring target volume or organs at risk and with standard plan approach. Dose prescription, fractionation, and treatment planning practices vary from site to site. Without imaging, dose must be prescribed in terms of fixed distances from a known reference, such as the applicator surface. Because of different anatomies of patients, this might lead to under-dosing of target and unnecessarily high-doses delivered to adjacent organs. Also, reliable recording of dose delivered is difficult. These various uncertainties related to standard planning and lack of imaging indicate a clear need for finding an optimal method of dose planning for vaginal cuff brachytherapy.

Material and methods: A study was conducted, in which 100 vaginal cuff brachytherapy patients' computed tomography (CT) images with applicator in situ were retrospectively analyzed to investigate target-area coverage and critical-organ doses. In addition, 28 patients' plans were re-planned with different planning approaches, to evaluate an optimal dose-planning strategy. From treatment plans, target coverage and organs-at-risk doses were assessed.

Results and conclusions: The analysis showed that, in order to cover distal part of the vaginal cuff, dose prescription should be a $10 \mathrm{~mm}$ from the tip of the applicator. Individualized image-based planning is recommended at least for first fraction. This would yield lower doses to the bladder. Rectum and sigmoid doses are not significantly affected by planning approach.

J Contemp Brachytherapy 2021; 13, 6: 687-693 DOI: https://doi.org/10.5114/jcb.2021.112120
\end{abstract}

Key words: vaginal cuff brachytherapy, brachytherapy, endometrial cancer, image-based brachytherapy planning.

\section{Purpose}

Endometrial cancer is the fourth most common malignancy among women in industrialized countries [1]. According to the World Health Organization (WHO), its' worldwide annual incidence reaches above 380,000, and it is responsible for nearly 90,000 cancer deaths a year [2]. Also, the incidence of endometrial cancer is rising, most likely because of population aging, obesity increasing, changes in reproduction trends, and perhaps, exposure to estrogens [1]. Regrettably, notwithstanding the improved treatment possibilities, survival rates have not improved over the years, where $20 \%$ of endometrial cancer patients still die of the disease [3].

The standard treatment option for endometrial cancer is surgery. Total removal of the uterus, cervix, ovaries, and fallopian tubes is the most common approach today [4]. The most common location for relapse after surgical operation is the vaginal cuff. To reduce local recurrence, adjuvant post-operative radiotherapy can be included in the treatment, although this has shown negligible effects on the overall likelihood of survival. Depending on the stage and grade of the disease and patient's characteristics, such as age and affected lymph nodes, adjuvant radiotherapy has been recommended in several reports [4-6]. In the PORTEC-1 study [7], the authors concluded that external beam radiotherapy (EBRT) should be avoided for low- and intermediate-risk patients in the interest of long-term quality of life related to bowel toxicity. In addition, the randomized PORTEC-2 study [8] proved that for intermediate- and high-risk patients, vaginal brachytherapy (VBT) is not inferior to EBRT and leads to fewer complications.

Vaginal brachytherapy is normally performed with a high-dose-rate (HDR) or pulsed-dose-rate (PDR) afterloader. Radiation is delivered to the patient via a vaginal applicator, and the diameter is adjusted to patient's anatomy. Applicators have either a single-central channel or several channels, although there is not a major difference between applicator types with regard to dose coverage of the target volume $[9,10]$.

Address for correspondence: Jan-Erik Palmgren, Department of Radiotherapy Kuopio University Hospital, Received: 24.02 .2021 P.O. Box 100, 70029 KYS, Finland, phone: +358-44-7113417, fax: +358-17-172936, 
Before radiation delivery, a treatment plan is made. This may be a standard plan or a patient-specific plan based on imaging; a standard plan is applied to every patient, without any imaging, or alternatively, the imaging may be used to record doses to organs at risk (OARs). Although 3D imaging and image-based planning is currently the standard treatment in radiotherapy clinics with external beam dose delivery, it has not been considered essential in VBT dose planning [5, 11, 12]. This stands in contrast to, for example, cervical cancer brachytherapy, for which treatment planning based on 3D magnetic resonance imaging (MRI) is highly recommended [13]. Because the vaginal wall conforms to applicator, target structure is considered to be more robust, giving the impression that contouring of clinical target volume (CTV) is not needed. Instead, the dose prescription is commonly defined at a certain depth from the applicator's surface. However, since patients' anatomy may vary naturally and because of surgical procedures, prescribing a dose without image-based planning may lead to inadequate coverage of the target volume [10], or unnecessarily high doses to OARs.

The GEC-ESTRO working group recommends imaging of patient with vaginal applicator in situ to verify applicator's placement and to determine doses to OARs [6], while the American Brachytherapy Society (ABS) has no recommendations on imaging or treatment planning [5], leaving that decision to brachytherapy centers. According to an ABS survey from 2014 [14], 73\% of brachytherapy centers create a treatment plan only for first fraction. A more recent paper by Martell et al. [15] states that $64 \%$ of centers use computed tomography (CT) for planning, and 33\% perform imaging for every fraction. There is no clear consensus on this topic, and practices differ, as several reviews demonstrated $[11,15,16]$. In our study, we analyzed four distinct planning approaches to investigate the optimal way of VBT planning.

Some research has examined the necessity of individualized fraction planning, with varying conclusions. Holloway et al. [17] assumed that a small within-patient variation in doses to OARs does not support reporting doses beyond initial fraction. Another paper compared individual-fraction and first-fraction-only planning, but did not decide which one is the better option [18]. More

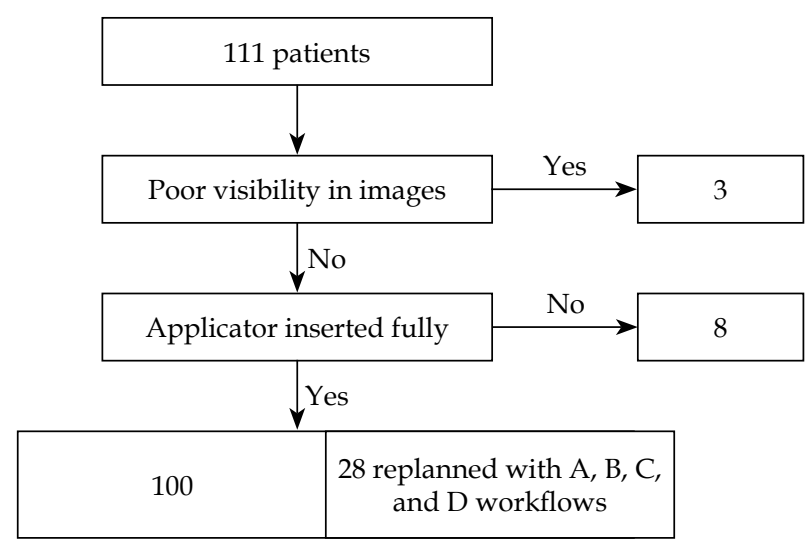

Fig. 1. Patient statistics recent articles $[19,20]$ have evaluated the need for imaging and re-planning between the first and other fractions, concluding that individualized planning is not needed and only patient with a previous radiotherapy might need imaging for all fractions. If brachytherapy is planned as a boost to EBRT, OAR's doses could be considerable higher and deviations in OAR's anatomy between fractions are more critical dose wise. Total doses might exceed recommended OARs' limits if brachytherapy treatment is not imaged and optimized according to treatment day situation. None of these papers, however, has addressed adequate dose coverage and dose prescription. In earlier work, target area has been studied [21] by looking at the lymphatic channel locations in the vaginal wall. Results of the study are consistent with our visual findings that vaginal-wall thickness in the lateral direction should be at least $5 \mathrm{~mm}$. However, imaging allows us to see that the wall varies in thickness at the vaginal stump. This leads one to ask whether we are covering the target area adequately with uniform $5 \mathrm{~mm}$ prescription depth.

\section{Material and methods}

This study included 111 consecutive post-operative endometrial cancer patients treated with CT-based VBT. Three patients were excluded because of inadequate CT visibility due to hip implants. With eight patients, the applicator was not inserted properly, leaving 100 patients eligible to participate. Patient statistics are presented in Figure 1. Of these 100 patients, 28 consecutive patients' treatment plans were re-planned, with four distinct planning approaches involving different dose prescriptions and planning methods, which resulted in 112 plans for the workflow analysis and 100 patients for the assessment of vaginal wall thickness.

For the analysis of target coverage's adequacy, vaginal wall/scar tissue thickness was measured from CT images. This was done independently by two experienced professionals, a gynecological oncologist and a medical physicist. Measurement was conducted from the first fraction image, from the tip surface of applicator to the visible vaginal wall/scar tissue (Figure 2). This was because in our experience, the vaginal-wall thickness differs most in that direction, on account of the scar tissue from the surgery and possible variations in applicator placement. To keep the same depth of insertion between fractions, applicator length outside of the patient was measured and maintained the same with every fraction. Interobserver variation was evaluated via analysis of inter-observer agreement (IOA) within the sets of measurements. Vaginal wall/scar tissue thickness, as measured, was divided into four categories, including $\leq 5 \mathrm{~mm}$, 5-10 $\mathrm{mm}, 10-15 \mathrm{~mm}$, and $>15 \mathrm{~mm}$.

In order to reveal how differences between dose-planning approaches affect OARs' doses, 28 patients' dose plans were re-planned in four separate ways.

The planning approaches were as follow:

A. An individualized image-based plan for the first fraction, with the plan copied for other fractions;

B. Every fraction being planned individually; 
C. A standard plan with a $5 \mathrm{~mm}$ uniform dose prescription from the surface of applicator;

D. A standard plan with a $10 \mathrm{~mm}$ dose prescription at the tip and $5 \mathrm{~mm}$ for the applicator's lateral direction (Figure 2).

With all approaches, a single-channel applicator was used, and dwell positions were activated according to prescription length, $1 \mathrm{~mm}$ apart. Dose points were added at the prescription distance from the applicator's surface, $10 \mathrm{~mm}$ from the tip of applicator and $5 \mathrm{~mm}$ at the side. Automatic optimization was used with a combination of manual and graphical methods for identifying dwell times.

Individualized planning was performed such that the prescription isodose line $\left(\mathrm{D}_{100 \%}\right)$ was adjusted to cover as well as possible the vaginal wall and the scar tissue, including possible 'dog-earing' at the tip of applicator, while avoiding $\mathrm{D}_{100 \%}$ to OARs. Dose prescription length was $1 / 3$ of the measured vaginal length, or at least $30 \mathrm{~mm}$ at minimum in all of the approaches.

Doses to $0.1 \mathrm{cc}$ volume $\left(\mathrm{D}_{0.1 \mathrm{cc}}\right)$ and $2 \mathrm{cc}$ volume $\left(\mathrm{D}_{2 \mathrm{cc}}\right)$ of OARs were recorded during the entire treatment. Fractional doses were converted to $2 \mathrm{~Gy}$ biological equivalent $\left(\mathrm{EQD}_{2}\right)$ doses using linear-quadratic (LQ) model, with $\alpha / \beta$ values of $3 \mathrm{~Gy}$ for the rectum and bladder, respectively [22].

Doses were added from three fractions, reflecting a total dose that a patient would receive to OARs under each planning approach. Statistical analysis was done for dosimetric parameters using Microsoft Excel ${ }^{\mathrm{TM}}$ calculations, with standard statistical analysis tools. To determine $p$-values for significance of differences, a one-direction ANOVA test was applied.

Additionally, differences in OARs' doses between fractions were analyzed to reveal how much OARs' doses change in the response of applicator placement and organ movement, with regard to each patient.

\section{Procedure}

A CT/MRI-compatible cylindrical plastic singlechannel treatment applicator (Elekta ${ }^{\mathrm{TM}}$ device) was inserted into the patient's vagina by a gynecologic oncologist for the first fraction, and by a radiation therapist for subsequent fractions. A standard insertion technique, described well in the literature [23, 24] was used. Applicator diameter was selected to fit the patient's anatomy. In connection with the first fraction, clinical examination of the vagina was conducted and vagina depth was measured to determine treatment length, which was used for all following fractions. To achieve replication of anatomy, a Foley-type urinary catheter was inserted into the bladder, and the bladder was filled with $100 \mathrm{cc}$ of saline water. This was done prior to imaging and repeated before every treatment fraction. The rectum was not prepared, unless large amounts of gas or feces were detected from images, in which case, the catheter was used to bleed the gas out, and imaging was repeated. Patient's imaging was done before every treatment fraction using a CT scanner (Toshiba Aquilion LB) from the vulva to the superior part of the bladder. A case-specific 3D treatment plan was prepared

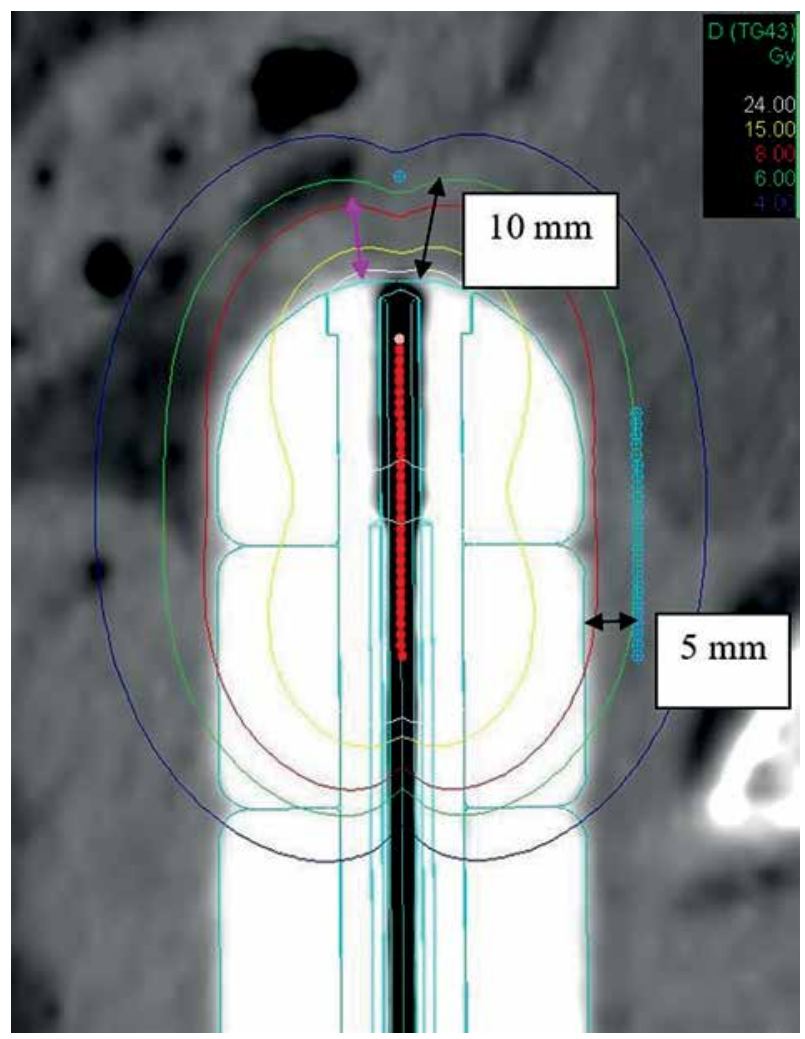

Fig. 2. Definition of the dose prescription: $10 \mathrm{~mm}$ at the tip of the applicator and $5 \mathrm{~mm}$ laterally from the surface of the applicator. The purple arrow shows how the vaginal wall/ scar tissue thickness was measured. Isodose lines (Gy): white 24 , yellow 15, red 8, green (prescription) 6, blue 4

with treatment-planning software (OncentraBrachy ${ }^{\mathrm{TM}}$ / Elekta $^{\mathrm{TM}}$ software) by a medical physicist for every fraction. The bladder, rectum, and sigmoid were contoured from the images as OARs. If the small bowel was near the treatment site, it was included into sigmoid structure.

All patients received VBT within the distal third of the vagina. The dose prescribed was $18 \mathrm{~Gy}$, over three fractions, with treatment every other day. None of the patients in the study received EBRT.

The study received the Kuopio University Hospital's ethics-board approval (No. 376).

\section{Results}

Independently, two professionals measured vaginal wall/scar tissue thickness at the tip of the applicator for the 100 included patients. The IOA calculated between individual measurements was $75 \%$. The average vaginal wall/scar tissue thickness was $7.0 \mathrm{~mm}$, with a standard deviation of $3.2 \mathrm{~mm}$, and minimum and maximum values were $1.6 \mathrm{~mm}$ and $17.1 \mathrm{~mm}$, respectively. The most prominent band was 5 to $10 \mathrm{~mm}$, representing more than $60 \%$ of the patients. Figure 3 shows the distribution of the thicknesses measured by each of the observers.

The patient-by-patient analysis showed that a uniform $5 \mathrm{~mm}$ prescription dose would cover the actual vaginal wall/scar tissue in only $31 \%$ of the patients, and a $10 \mathrm{~mm}$ prescription would do the same with $86 \%$. 


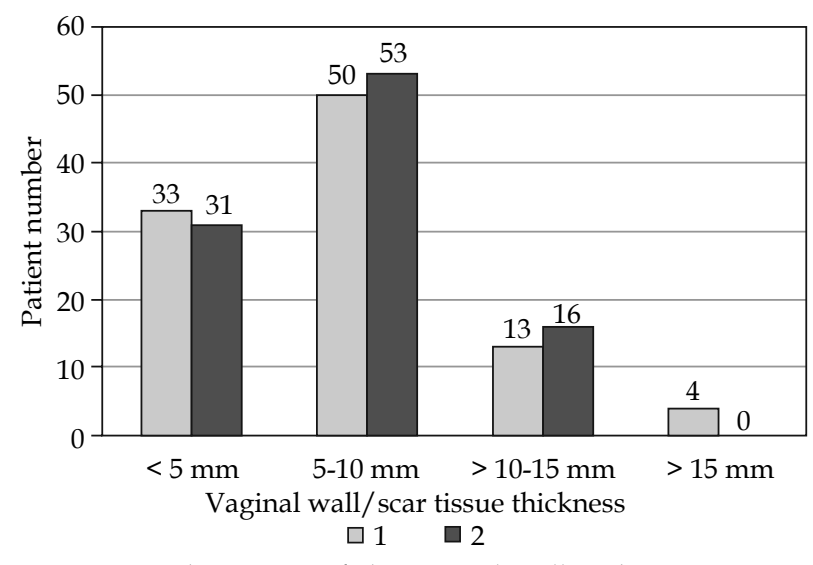

Fig. 3. A histogram of the vaginal wall and scar tissue thickness measured from the tip of the applicator. The two bars are from observer 1 and observer 2

In OAR's dose analysis, a two standard-plan approaches $(C$ and $D)$ produced statistically the highest doses to the bladder $(p<0.001)$, while the lowest bladder doses were achieved with approach $\mathrm{B}$. This was true for both the $\mathrm{D}_{0.1 \mathrm{cc}}$ and the $\mathrm{D}_{2 \mathrm{cc}}$ doses. For standard-planning approach $\mathrm{D}$, relative to individualized planning (B), the $\mathrm{D}_{2 \mathrm{cc}}$ and $\mathrm{D}_{0.1 \mathrm{cc}}$ values were higher, at $19.45 \mathrm{~Gy}$ and $26.11 \mathrm{~Gy}$, as opposed to $16.23 \mathrm{~Gy}$ and $20.12 \mathrm{~Gy}$, respectively For the rectum and sigmoid colon, there were no statistically significant differences between any of the planning approaches. Table 1 presents the results.

To estimate total biological doses to OARs, the dose values were converted to 2 Gy equivalents by using an
LQ model with $\alpha / \beta=3 \mathrm{~Gy}$. For the total dose to OARs of each patient, the three fractional doses were summed on the presumption that $0.1 \mathrm{cc}$ and $2 \mathrm{cc}$ volumes' locations did not change between fractions (worst-case assumption). The values are presented in Table 2.

Variation of OARs' doses between fractions for each patient was estimated by calculating the patient-specific standard deviation. This analysis revealed a small deviation, about $0.5 \mathrm{~Gy}$ in $\mathrm{D}_{2 \mathrm{cc}}$ values. The number was especially low with respect to the bladder and imagebased planning (approaches A and B), reaching its' lowest with approach A, i.e., $0.18 \mathrm{~Gy}$. The largest deviations were recorded with $\mathrm{D}_{0.1 \mathrm{cc}}$ values for the bladder and sigmoid colon and with the planning approach D at around $1 \mathrm{~Gy}$. The detailed values are presented in Figure 4.

\section{Discussion and conclusions}

Without 3D soft tissue imaging, such as CT or MRI, there is a risk of not detecting relevant anatomical information from the target area, for example, scar tissue at the deep end of the vaginal stump, which would be considered a part of CTV. Imaging can also reveal whether the applicator has been fully inserted, reaching the vaginal stump. Furthermore, relevant OARs' location of the urinary bladder, rectum, and sigmoid colon/bowel, change between the fractions, and may end up being close to the applicator. That would increase doses to OARs [20, 25, 26], or at least received doses may be left uncertain. From the perspective of different workflows and application itself, there is very limited information available that present the optimum approach $[27,28]$.

Table 1. Average OARs' doses (Gy) by planning approach ( $p$-values calculated from individual plans, $n=28$ )

\begin{tabular}{lcccccc} 
& Bladder $\mathrm{D}_{0.1 c c}$ & $\begin{array}{c}\text { Bladder } \\
\mathrm{D}_{2 c c}\end{array}$ & Rectum $\mathrm{D}_{0.1 c c}$ & $\begin{array}{c}\text { Rectum } \\
\mathrm{D}_{2 c c}\end{array}$ & $\begin{array}{c}\text { Sigmoid } \\
\mathrm{D}_{0.1 c c}\end{array}$ & $\begin{array}{c}\text { Sigmoid } \\
\mathrm{D}_{2 c c}\end{array}$ \\
\hline $\mathrm{A}$ & 20.7 & 16.7 & 16.9 & 12.6 & 11.7 & 8.1 \\
\hline $\mathrm{B}$ & 20.1 & 16.2 & 17.2 & 12.7 & 12.0 & 8.2 \\
\hline $\mathrm{C}$ & 21.2 & 17.6 & 18.0 & 13.7 & 12.1 & 8.4 \\
\hline $\mathrm{D}$ & 26.1 & 19.5 & 18.0 & 13.6 & 14.4 & 9.7 \\
\hline$p$-value & 0.00 & 0.00 & 0.51 & 0.50 & 0.17 & 0.27
\end{tabular}

Table 2. Average and maximum biological 2 Gy equivalent OARs' doses (Gy) by planning approach

\begin{tabular}{lcccccc} 
& $\begin{array}{c}\text { Bladder } \\
\mathrm{D}_{0.1 c c}\end{array}$ & $\begin{array}{c}\text { Bladder } \\
\mathrm{D}_{2 c c}\end{array}$ & $\begin{array}{c}\text { Rectum } \\
\mathrm{D}_{0.1 c c}\end{array}$ & $\begin{array}{c}\text { Rectum } \\
\mathrm{D}_{2 c c}\end{array}$ & $\begin{array}{c}\text { Sigmoid } \\
\mathrm{D}_{0.1 c c}\end{array}$ & $\begin{array}{c}\text { Sigmoid } \\
\mathrm{D}_{2 c c}\end{array}$ \\
\hline Average & & & & & & \\
\hline $\mathrm{A}$ & 41.0 & 28.9 & 28.9 & 18.1 & 31.5 & 9.2 \\
\hline $\mathrm{B}$ & 39.0 & 27.2 & 29.8 & 18.1 & 16.8 & 9.2 \\
\hline C & 43.0 & 31.5 & 24.0 & 20.2 & 16.8 & 9.7 \\
\hline D & 61.1 & 37.0 & 24.0 & 20.2 & 22.5 & 11.9 \\
\hline Maximum & & & & & 35.2 & 18.1 \\
\hline A & 52.8 & 34.2 & 42.0 & 27.2 & 39.0 & 19.5 \\
\hline B & 50.5 & 32.4 & 45.1 & 28.9 & 42.0 & 27.2 \\
\hline C & 66.1 & 42.0 & 62.3 & 41.0 & 62.3 & 28.9
\end{tabular}


Bladder $0.1 \mathrm{cc}$

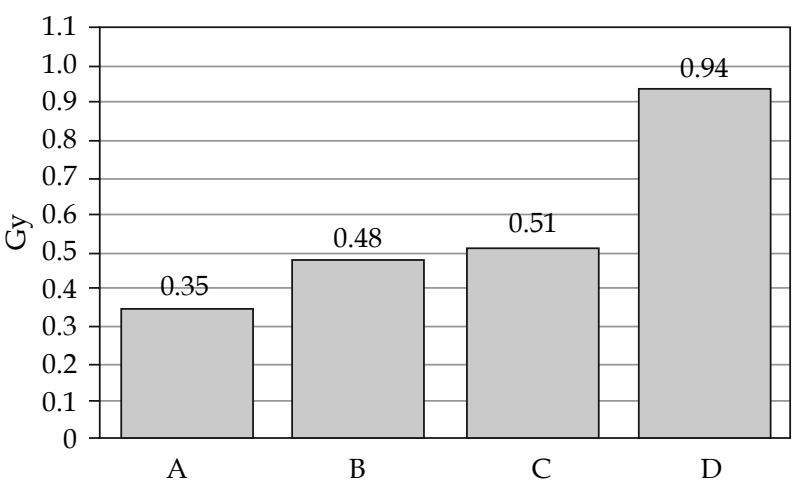

Rectum $0.1 \mathrm{cc}$

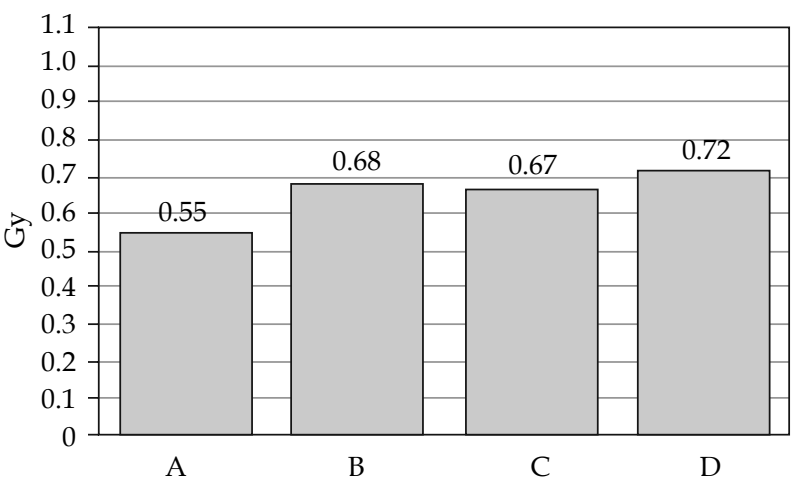

Sigmoid $0.1 \mathrm{cc}$

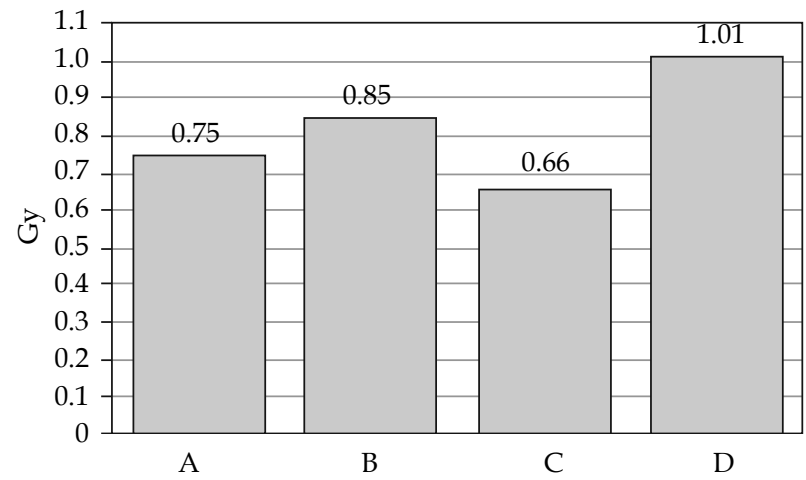

Bladder $2 \mathrm{cc}$

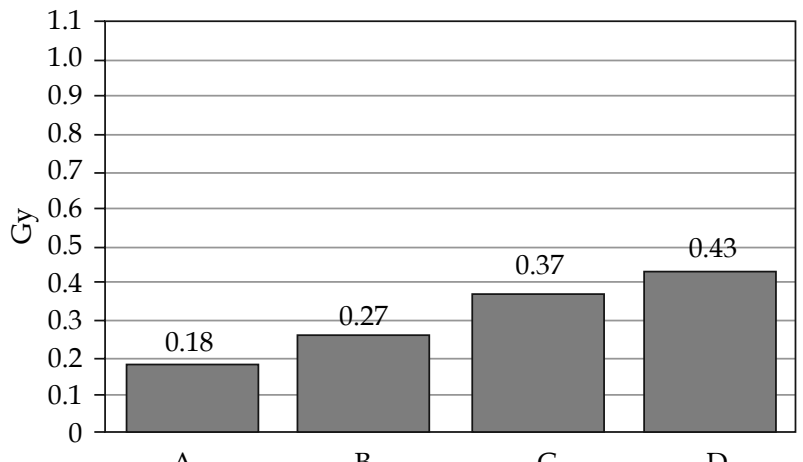

A

Rectum 2 cc

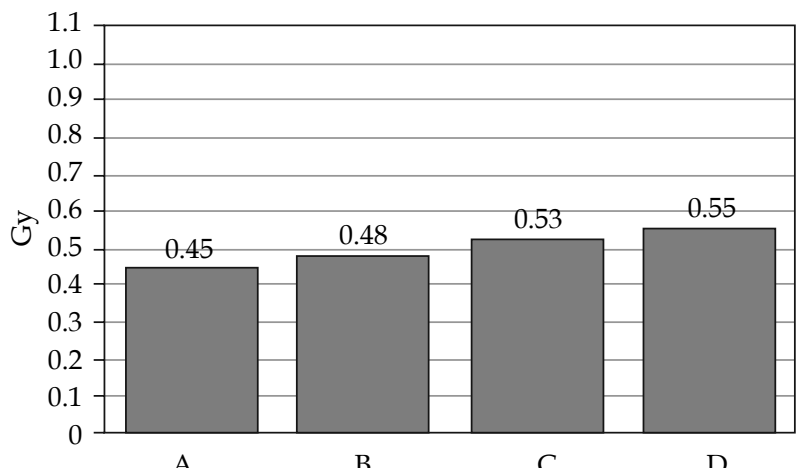

Sigmoid 2 cc

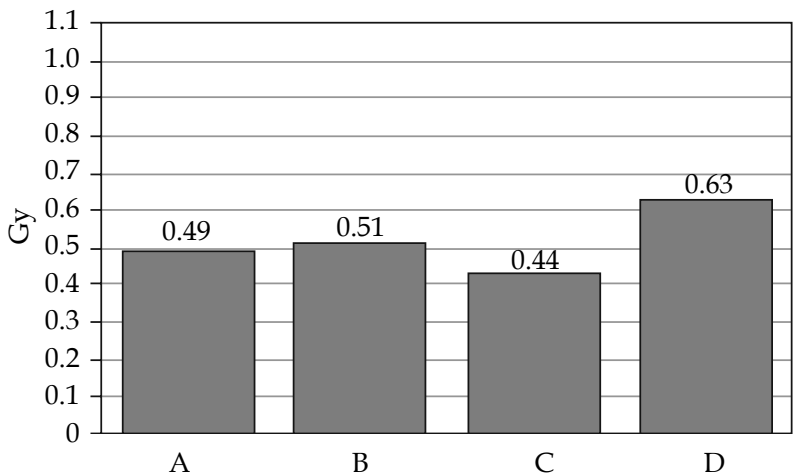

Fig. 4. Standard deviation of doses between fractions, by planning approach

Challenges of imaging and planning with every treatment fraction include more time needed and human resources. Also, cumulative doses to OARs from VBT might be insignificant considering morbidity. On the other hand, these patients have a good prognosis and could live a long life; therefore, the possibility of later irradiation within the same area have to be considered. If information on doses received by OARs from VBT are unavailable, considering new plan is difficult.

To determine the optimal way of planning VBT, one must assess the target coverage and OARs doses for alternative plan approaches. Normally, the doses to OARs are not an issue in VBT, because the total dose is low and dose gradient is steep; however, if an organ is in close proximity to the applicator, the doses increase vastly and the point doses could be especially high. The afore-men- tioned issues and previous radiotherapy may affect OARs' dose limits.

This study shows the importance of image-based planning in prophylactic vaginal brachytherapy, with regard to achieving good target dose coverage. Defining the target with a standard fixed distance from the applicator does not suffice for covering all individual anatomical variations in CTV. To reach an adequate target coverage, one can contour the target from the images and treat on this basis, or increase the fixed prescription distance. The findings of the study indicate that a $10 \mathrm{~mm}$ prescription distance at the distal end of the vagina should cover the treatment target for more than $80 \%$ of patients.

It has been established that CT may exhibit limitations with soft tissue contrast and that inter-observer variation may affect target definition [29]. Therefore, we had two 
experienced professionals to measure the thickness of the target volume. From the 100 patients, the tissue-depth classification was very similar, even though IOA for the measured values was only $75 \%$ (Figure 3 ). Most cases of disagreement involved greatest distances (above $15 \mathrm{~mm}$ ). The vaginal wall/scar tissue being so thick may suggest that the applicator was not inserted fully or was too wide to address the bottom of the vagina properly. From the sample of 111 patients, we found that the applicator was not fully inserted in at least eight cases. Both of these findings highlight the need of imaging-based dose planning.

Using vaginal mold type applicator, which is created according to patient's vagina anatomy, it may be possible to avoid incomplete insertions. In a recent study [30], mould applicator was compared to standard cylinder and found to be superior regarding dose coverage of CTV and also OAR's saving (the bladder).

Our OARs' analysis revealed that the option B (planning for each fraction) led to the lowest dose to the bladder, whereas approach A (planning for the first fraction) led to the lowest rectum and sigmoid doses, with difference being statistically significant only for bladder doses. It was expected that both of the planning approaches yielding lower values were image-based since the ability to see OARs created a possibility of sparing them in dose planning whenever possible, without compromising target coverage.

However, when we look at average total $\mathrm{D}_{2 c c} \mathrm{EQD}_{2}$ doses, we see reasonably low values from all the planning approaches (Table 2): the highest bladder dose was 37.0 Gy, with the corresponding rectum and sigmoid doses being 20.2 Gy and 11.9 Gy, respectively. Even the maximum $\mathrm{EQD}_{2} \mathrm{D}_{2 \mathrm{cc}}$ doses (50.5 Gy, 41.0 Gy, and 28.9 Gy, respectively) were well below the limit of 70-80 Gy, a maximum value referenced in recommendations for image-guided cervical cancer brachytherapy [13].

As for $\mathrm{D}_{0.1 \mathrm{cc}}$ values, the average bladder dose was largest with standard planning (D) at $61.1 \mathrm{~Gy}$, and the maximum was 108.0 Gy. The rectum and sigmoid values showed a similar but more modest pattern, with the maximum dose being $62.3 \mathrm{~Gy}$ for both $\mathrm{C}$ and D approaches.

In this study, we also considered whether imaging for just the first fraction would be safe regarding OARs' doses. The results show dose deviation between fractions to be modest. The standard deviation was very low for $\mathrm{D}_{2 \mathrm{cc}}$ doses $(0.5 \mathrm{~Gy})$ and modest for $\mathrm{D}_{0.1 \mathrm{cc}}$ doses (under $1.0 \mathrm{~Gy}$ ). This indicates that it is acceptable to image the patient and plan only for the first fraction, then copy the created plan to subsequent fractions. Measuring the applicator depth on the first fraction instance and replicating this depth for the rest of the fractions also assures that the applicator placement is adequate. First-fraction-only imaging should save imaging and personnel resources, therefore, make the treatment process shorter for the patient.

Our results show that, in order to be able to cover the vaginal wall/scar tissue in the distal portion of the vagina for $82 \%$ of patients, the dose for VBT should be prescribed for $10 \mathrm{~mm}$ distally from the surface of the applicator. This can be done safely with respect to OARs by means of imaging-guided individualized planning workflow, with only first fraction planned. Of the planning approaches studied, this one would yield the lowest dose to the bladder, for both $0.1 \mathrm{cc}$ and $2 \mathrm{cc}$ volumes. The rectum and sigmoid doses did not differ significantly between any of the planning choices.

If the prescription depth is increased from 5 to $10 \mathrm{~mm}$ distally, the dose received by the bladder increases the most. However, the total maximum $\mathrm{EQD}_{2}$ values are still well below the recommended dose-limit levels. If patients would have received EBRT at the pelvic area, OAR's dose limits could be violated. This issue needs to be investigated separately and was not addressed in this study.

With imaging-based individualized planning, one can visualize the intended target volume in relation to dose distribution, which allows the verification of adequate target coverage. This should be the fundamental requirement for any modern radiotherapy dose planning approach.

\section{Disclosure}

The authors report no conflict of interest.

\section{References}

1. Ferlay J, Colombet M, Soerjomataram I et al. Estimating the global cancer incidence and mortality in 2018: GLOBOCAN sources and methods. Int J Cancer 2019; 144: 1941-1953.

2. WHO International Agency for Research on cancer. World cancer statistics. Available at https://www.iarc.fr/cards_ page/world-cancer-report/. Accessed May 2019.

3. Siegel RL, Miller KD, Jemal A. Cancer Statistics, 2019. CA Cancer J Clin 2019; 69: 7-34.

4. Brooks RA, Fleming GF, Lastra RR et al. Current recommendations and recent progress in endometrial cancer. CA Cancer J Clin 2019; 69: 258-279.

5. Nag S, Erickson B, Parikh S et al. The American Brachytherapy Society recommendations for high-dose-rate brachytherapy for carcinoma of the endometrium. Int J Radiat Oncol Biol Phys 2000; 48: 779-790.

6. Hoskin P, Paulsen T, Nout R et al. The GEC ESTRO Handbook of Brachytherapy, Part II: Clinical Practice, 17 Endometrial Cancer 2016, Version 1-25/04/: 3-20.

7. Nout RA, van de Poll-Franse LV, Lybeert ML et al. Longterm outcome and quality of life of patients with endometrial carcinoma treated with or without pelvic radiotherapy in the Post-Operative Radiation Therapy in Endometrial Carcinoma 1 (PORTEC-1) trial. J Clin Oncol 2011; 29: 1692-1700.

8. Nout RA, Smit VT, Putter H et al. Vaginal brachytherapy versus pelvic external beam radiotherapy for patients with endometrial cancer of high-intermediate risk (PORTEC-2): an open-label, non-inferiority, randomized trial. Lancet 2010; 9717: 816-823.

9. Zhang H, Gopalakrishnan M, Lee P et al. Dosimetric impact of cylinder size in highdose rate vaginal cuff brachytherapy (VCBT) for primary endometrial cancer. J Appl Clin Med Phys 2016; 17: 262-272.

10. Li S, Aref I, Walker E et al. Effects of prescription depth, cylinder size, treatment length, tip space, and curved end on doses in high-dose-rate vaginal brachytherapy. Int J Radiat Oncol Biol Phys 2007; 67: 1268-1277.

11. Sabater S, Andres I, Lopez-Honrubia V et al. Vaginal cuff brachytherapy in endometrial cancer - a technically easy treatment? Cancer Manag Res 2017; 9: 351-362. 
12. Guy CL, Fields EC, Quinn BA et al. The vaginal cylinder: misunderstood, misused, or trivial? An in-depth dosimetric and multiinstitutional outcome investigation. Brachytherapy 2019; 18: 763-770.

13. Pötter R, Haie-Meder C, Van Limbergen E et al. Recommendations from gynaecological (GYN) GEC ESTRO working group (II): concepts and terms in 3D imagebased treatment planning in cervix cancer brachytherapy - 3D dose volume parameters and aspects of 3D image-based anatomy, radiation physics, radiobiology. Radiother Oncol 2006; 78: 67-77.

14. Harkenrider MM, Grover S, Erickson BA et al. Vaginal brachytherapy for postoperative endometrial cancer: 2014 survey of the American Brachytherapy Society. Brachytherapy 2016; 15: 147-152.

15. Martell K, Doll C, Barnes EA et al. Radiotherapy practices in postoperative endometrial cancer: a survey of the ABS membership. Brachytherapy 2019; 18: 741-746.

16. Harkenrider MM, Block AM, Alektiar KM et al. American Brachytherapy Task Group Report: Adjuvant vaginal brachytherapy for early-stage endometrial cancer: a comprehensive review. Brachytherapy 2017; 16: 95-108.

17. Holloway CL, Macklin EA, Cormack RA et al. Should the organs at risk be contoured in vaginal cuff brachytherapy? Brachytherapy 2011; 10: 313-317.

18. Symon Z, Menhel J, Alezra D et al. Individual fraction optimization vs. first fraction optimization for multichannel applicator vaginal cuff high-dose-rate brachytherapy. Brachytherapy 2006; 5: 211-215.

19. Corso CD, Jarrio C, Nunnery EW et al. Dosimetric and cost comparison of first fraction imaging versus fractional re-imaging on critical organ dose in vaginal cuff brachytherapy. Pract Radiat Oncol 2013; 3: 256-262.

20. Zhou J, Prisciandaro J, Choonik L et al. Single or multi-channel vaginal cuff highdose-rate brachytherapy: is replanning necessary prior to each fraction? Pract Radiat Oncol 2014; 4 : 20-26.

21. Choo J, Scudiere J, Bitterman P et al. Vaginal lymphatic channel location and its implication for intracavitary brachytherapy radiation treatment, Brachytherapy 2005; 4: 236-240.

22. Fowler J. The linear-quadratic formula and progress in fractionated radiotherapy. $\mathrm{Br}$ J Radiol 1989; 62: 679-694.

23. Harkenrider MM, Block AM, Siddiqui ZA et al. The role of vaginal cuff brachytherapy in endometrial cancer. Gynecol Oncol 2015; 136: 365-372.

24. Small W Jr, Beriwal S, Demanes DJ et al. American Brachytherapy Society consensus guidelines for adjuvant vaginal cuff brachytherapy after hysterectomy. Brachytherapy 2012; 11: 58-67.

25. Ozdemir Y, Dolek Y, Onal C et al. Effect of vaginal cylinder position on dose distribution in patients with endometrial carcinoma in treatment of vaginal cuff brachytherapy. J Contemp Brachytherapy 2017; 9: 230-235.

26. Sabater S, Sevillano MM, Arenas M et al. Reduction of rectal doses by removing of gas in the rectum during vaginal cuff brachytherapy. Srahlenter Onkol 2013; 189: 951-956.

27. Gruhl JF, Zheng D, Longo JL et al. Comparing organ-at-risk doses for high-dose-rate vaginal brachytherapy between three different planning workflows. Brachytherapy 2017; 16: 373-377.

28. Iatì G, Pontoriero A, Mondello $S$ et al. Three-dimensional treatment planning for vaginal cuff brachytherapy: dosimetric effects on organs at risk according to patients position. Brachytherapy 2014; 13: 568-571.

29. Chapman C, Prisciandaro JI, Maturen KE et al. MRI-based evaluation of the vaginal cuff in brachytherapy planning: are we missing the target? Int J Radiat Oncol Biol Phys 2016; 95: 743-750
30. Jreij M, Kattar ZAL, Charara J. Comparison between Stump Cylinder and custom mold, effect of the shape of the applicator on the dose distribution. Cancer Radiother 2020; 24: 106-113. 